\title{
LAPLACIANS AND THE CHEEGER CONSTANTS FOR DISCRETE DYNAMICAL SYSTEMS
}

\author{
SARA FERNANDES ${ }^{1}$, CLARA GRÁCIO ${ }^{1}$ AND J. SOUSA RAMOS ${ }^{2}$
}

We consider discrete laplacians for iterated maps on the interval and examine their eigenvalues. We have introduced a notion of conductance (Cheeger constant) for a discrete dynamical system, now we study their relations with the spectrum. We compute the systoles and the first eigenvalue of some families of discrete dynamical systems.

2000 MCS Primary 37B10, 05C35; Secondary 37E05, 37E15

Keywords: Discrete Laplacian, Cheeger constant, discrete dynamical system, systole

Dedicated to the Memory of Bernd Aulbach

\section{Introduction}

Our subject is the study of discrete dynamical systems arising from the iterates of a map in the interval and our main concern is to search for invariants which could differentiate systems with the same topological entropy. In this paper is made a strong connection between a geometric point of view and a discrete dynamical system point of view. We explore the connections Cheeger constant/conductance, Laplace-Beltrami operator/discrete laplacian and systoles and isoperimetric inequalities in both contexts.

We begin with the geometric motivation, see Grácio-Sousa Ramos ${ }^{5}$.

The idea of a Riemann surface is a central one in mathematics, and appears in such seemingly diverse areas as low dimensional topology, algebraic, differential and hyperbolic geometry, complex analysis, group theory and even number theory. This paper concerns compact Riemann surfaces endowed with a hyperbolic metric. It is possible to describe it in some ways, these include its representation as an algebraic curve; as a period matrix; as a Schottky group; as a Fuchsian group and as a hyperbolic manifold, in particular, using Fenchel-Nielsen (F-N) coordinates. It is exactly this last way that we will use, Riemann surface theory based on closed geodesics using Fenchel-Nielsen (F-N) coordinates. This geometry has always played 
an important part in the study of Riemann surfaces. There are directly related to very active subjects such as spectral theory, Teichmuller spaces and number theory. One of the most natural objects in this context are the systoles, the shortest closed geodesics of a Riemann surface. They provide perfect analogues to classical problems of lattice sphere packings. In this paper we will introduce and evaluate explicitly the systoles in discrete dynamical systems.

Given a compact Riemannian manifold $M$, the Laplace-Beltrami operator $\Delta$ on functions on $M$ is an elliptic operator with discrete spectrum

$$
0=\mu_{0}<\mu_{1}<\mu_{2}<\ldots<\mu_{k} \ldots \rightarrow \infty \text {. }
$$

Whereas in the case of surfaces of genus 0 or 1 the explicit computation is possible, this is not the case for surfaces of higher genus. For surfaces of genus $g=2$ we can compute numerically the geodesic length spectrum of $M$ (lengths of closed geodesics)

$$
0=\ell_{0}<\ell_{1}<\ell_{2}<\ldots<\ell_{k} \ldots \rightarrow \infty
$$

and using this spectrum to computing the $\mu_{i}, i \in \mathbb{N}$. These surfaces can be obtained by gluing together pairs of pants with no twists on the boundary components. This corresponds to Riemann surfaces in Teichmüller space for which the Fenchel-Nielsen $(\mathrm{F}-\mathrm{N})$ coordinates are of the form $\left(\ell\left(\gamma_{1}\right), \ell\left(\gamma_{2}\right), \ell\left(\gamma_{3}\right), 0,0,0\right)$, where $\ell\left(\gamma_{i}\right)$ are the lengths of the three geodesics on the surface.

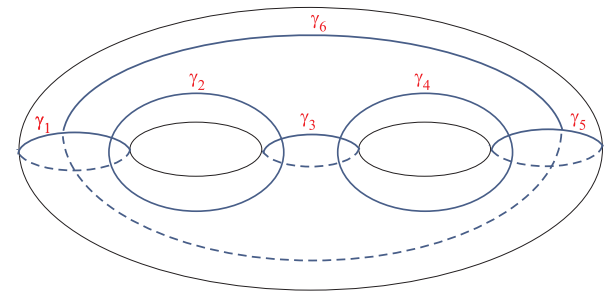

Figure 1. Bitorus: closed Riemann surface of genus 2.

One of the possible ways to describe a closed Riemann surface $\mathrm{M}$ of genus $g$ are the F-N coordinates, that consists in the lengths and twists of $3 g-3$ disjoint simple closed geodesics. This space of coordinates is homeomorphic 
to the Teichmüller space and the Teichmüller modular group acts on any such space of F-N coordinates as a group of algebraic diffeomorphisms.

In this context we can state the definition of the Cheeger constant, see Brooks ${ }^{4}$.

Definition 1.1. Given a Riemannian manifold $M$ (compact, no boundary), define the Cheeger constant by

$$
h(M)=\inf _{X} \frac{\operatorname{area}(X)}{\min (\operatorname{vol}(A), \operatorname{vol}(B))},
$$

where the infimum is over all compact separating hypersurfaces in $M$ ( $A$ and $B$ are the resulting disconnected sets).

A basic model for the discrete context is a graph $G=(V, E)$ consisting of a set $V$ of vertices and a prescribed set $E$ of pairs of vertices. For a subset $X$ of vertices in $G$, the volume $\operatorname{vol}(X)$ is defined by

$$
\operatorname{vol}(X)=\sum_{v \in X} d_{v}
$$

where $d_{v}$ is the degree of $v$. We denote the volume of $G$ by $\operatorname{vol}(G)=\sum_{v} d_{v}$ and the edge boundary is

$$
\partial(X)=\{\{x, y\} \in E: x \in X, y \in V \backslash X\} .
$$

Definition 1.2. The Cheeger constant of a graph $G$ with vertex set $V$ is defined to be

$$
h(G)=\inf _{X} \frac{|\partial(X)|}{\min (\operatorname{vol}(X), \operatorname{vol}(V \backslash X))},
$$

where the infimum is over all subsets $X$ of the vertex set and $|\partial(X)|$ is the number of edges joining the vertices of $X$ with vertices of $V \backslash X$, (See Bollobás ${ }^{3}$ for details). The smallest non-trivial eigenvalue $\mu_{1}$ of the Laplacian of a graph is closely related to the isoperimetric Cheeger constant. The Cheeger inequality for a graph $G$ states that

$$
2 h(G) \geq \mu_{1} \geq \frac{h(G)^{2}}{2} .
$$

Related with this kind of constants, the isoperimetric constants, is another geometric quantity, the systole, which has also a correspondent in graph theory:

In 1972 Marcel Berger defined metric invariants that capture the "size" of $k$-dimensional homology of a manifold. He asked if these invariants can 
be constrained by the volume. The invariant that captures the notion of the "size" is the systole, see Berger"

Definition 1.3. A systole of a Riemann surface $M$ of genus $g$ is the length of the shortest closed geodesic of $M$.

As usual there are the graph-theoretical analogue of this notion. One of our interest is to pass back and forth between the geometric and graphtheoretic pictures. Comparing various results may be an important source of insight.

After the geometric motivation we present now the dynamical settings. Consider a discrete dynamical system described by the iterates of a map $f$ in the interval. Suppose that $f$ is a Markov map, that is, a piecewise monotonic transformation that maps each interval of the partition onto a union of intervals of the partition. Let $A_{f}$ be the transition matrix associated to this system and let $P$ be the corresponding weighted stochastic matrix (see Parry $\left.{ }^{11}\right)$.

A row vector $\pi \in \mathbb{R}^{n}$ is a stationary distribution of the system with weighted transition matrix $P$ if and only if

a) $\pi(i) \geq 0$, for all $i \in V$;

b) $\sum_{i \in V} \pi(i)=1$;

c) $\pi=\pi P$.

A Markov chain with transition matrix $P$ is said to be ergodic if it has a stationary distribution. It is known that any finite, irreducible Markov chain is ergodic.

To each such dynamical system, there is an underlying graph $G$, whose vertex set $V$ is the set of intervals of the Markov partition and whose edges $i j$ correspond to non-zero entries $a_{i j}$ of the transition matrix $A_{f}$, also denoted in the graph context as $A(G)$.

Denote by $\{1,2, \ldots, n\}$ the set of vertices and by $e=(i j)$ the edge that joins the vertex $i$ with the vertex $j$. We say that $i$ is the starting point of $e$ and that $j$ is the end point of $e$. Let $w_{(i j)}=\pi_{i} P_{i j}$ the weight of the edge and let $\left(G_{f}, w\right)$ be the weighted graph associated to $f$. The volume of $\left(G_{f}, w\right)$ denoted by $\operatorname{vol}\left(G_{f}, w\right)$ is the sum of the weight of its edges

$$
\operatorname{vol}\left(G_{f}, w\right)=\sum_{e \in E} w(e) .
$$

The systole of $\left(G_{f}, w\right)$ is defined by

$$
\operatorname{sys}_{w}\left(G_{f}\right)=\inf \left\{p_{w}(c): c \text { is a non-loop cycle of } G_{f}\right\}
$$


where $p_{w}(c)$ is the product of the weights of its edges, compare to Balacheff ${ }^{1}$.

The geometric quantities we've seen in the geometry's motivation can be viewed as quantities in graph theory, and so, as quantities of a discrete dynamical system.

\section{Discrete Laplacian and Conductance}

Let $D(G)=\operatorname{diag}\left(d_{i}: i\right.$ is in $\left.V\right)$ be the diagonal matrix indexed by $V$ and with vertex degrees in the diagonal, that is, $d_{i i}$ is the number of edges beginning or ending in the vertex $i$.

Define the Laplace matrix of $G$ by

$$
\Delta(G)=D(G)-A(G) .
$$

It is easy to see that $\Delta(G)$ satisfy the relation

$$
\Delta(G)=Q Q^{T},
$$

where $Q$ is the incidence matrix with columns indexed by the edges and rows indexed by the vertices, defined as follows

$$
q_{i e}=\left\{\begin{array}{l}
-1 \text { if } i \text { is the starting point of } e \\
+1 \text { if } i \text { is the end point of } e \\
0 \text { if } i \text { is not incident on } e
\end{array},\right.
$$

see Mohar ${ }^{9}$. Note that in the construction of the incidence matrix $Q$ we had to chose arbitrarily an orientation and ignore the loops. In fact in the case of an unoriented graph it will make no difference.

The case we are concerned with in this paper corresponds to an oriented and weighted graph, i.e., we have no longer a symmetric transition matrix. Anyway we will define the Laplacian in a such way that the relation (1) will be satisfied and the Laplacian will be still a symmetric matrix (see Fernandes-Sousa Ramos ${ }^{6}$ )

$$
\Delta(G)=D(G)-\left(A+A^{T}\right)
$$

where $D(G)=\operatorname{diag}\left(d_{i i}: i\right.$ is in $\left.V\right)$, with

$$
d_{i i}=\sum_{j=1}^{n} a_{i j}+\sum_{j=1}^{n} a_{j i}
$$

is the matrix degree, or, for a weighted version

$$
\Delta^{*}(G)=D^{*}(G)-\left(P+P^{T}\right),
$$


where $D^{*}(G)=\operatorname{diag}\left(d_{i i}^{*}: i\right.$ is in $\left.V\right)$, with

$$
d_{i i}^{*}=\sum_{j=1}^{n} p_{i j}+\sum_{j=1}^{n} p_{j i}
$$

Now we define the conductance of a discrete dynamical system as follow.

Definition 2.1. The conductance of a discrete dynamical system with stationary distribution $\pi$ and stochastic transition matrix $P$ is defined by

$$
\Phi=\min _{\substack{\varnothing \neq U \subset V \\ 0 \neq|U| \leq \frac{1}{2}}} \frac{\sum_{i \in U, j \in V \backslash U} \pi_{i} P_{i j}}{\sum_{i \in U} \pi_{i}} .
$$

The conductance may be viewed as a weighted version of edge expansion of the graph underlying the Markov chain. For a fixed $U$, the quotient in the definition is just the conditional probability that the chain in equilibrium escapes from the subset $U$ of the state space in one step, given that it is initially in $U$. Thus $\Phi$ measures the ability of the system to escape from any small region of the state space, and hence to make rapid progress to the stationary distribution. It is used to bound the mixing rate of a discrete dynamical system, see Bollobás ${ }^{3}$.

Let's observe the behavior of certain families of maps. These maps are piecewise linear models, semiconjugated to quadratic and cubic maps.

We begin with the definition of the tent map. Let $I=[0,1]$, the unit interval, we consider a family of unimodal maps $f_{s}: I \rightarrow I$ defined by

$$
f_{s}(x)=\left\{\begin{array}{lll}
s x & \text { if } & 0<x<\frac{1}{2} \\
-s x+s & \text { if } & \frac{1}{2}<x<1
\end{array} .\right.
$$

These are Markov maps for certain $s \in(1,2)$. Now we define a family of bimodal maps. Consider $f_{s, e}: I \rightarrow I$ defined by

$$
f_{s, e}(x)= \begin{cases}s x & \text { if } 0<x<c_{1} \\ -s x+e & \text { if } c_{1}<x<c_{2} \\ s x+1-s & \text { if } c_{2}<x<1\end{cases}
$$

where $c_{1}=e / 2 s$ and $c_{2}=(e+s-1) / 2 s$. These are Markov maps for certain $s \in(1,3)$.

With this structure we are able to codify the trajectories using the symbolic dynamics in both cases. As usual for the unimodal case we use 
the alphabet $\{L, C, R\}$ and the rule

$$
\operatorname{ad}(x)=\left\{\begin{array}{l}
L \text { if } x<1 / 2 \\
C \text { if } x=1 / 2 \\
R \text { if } x>1 / 2
\end{array}\right.
$$

to codify the addresses of the trajectory of the critical point $c=1 / 2$, attributing the correspondent symbol to the iterates of $c$. For the bimodal case, denote by $L$ (left), $M$ (middle) and $R$ (right) the three laps of the interval $I$ and by $A$ and $B$ the two critical points according to a similar rule:

$$
L<A<M<B<R .
$$

For this case we need the sequences of both critical points $\left\{f^{i}\left(c_{1}\right)\right\}$ and $\left\{f^{i}\left(c_{2}\right)\right\}$, with $i \in \mathbb{N}$, the pair of kneading sequences, to have the topological classification of the maps by their trajectories (in true topological semicongugacy), see Milnor-Thurston ${ }^{10}$.

Our results are strongly supported by the observation of the tree with the trajectories of the unimodal family, ordered according to the topological entropy, see Lampreia et $a .^{7}$ for details about the tree of unimodal maps.

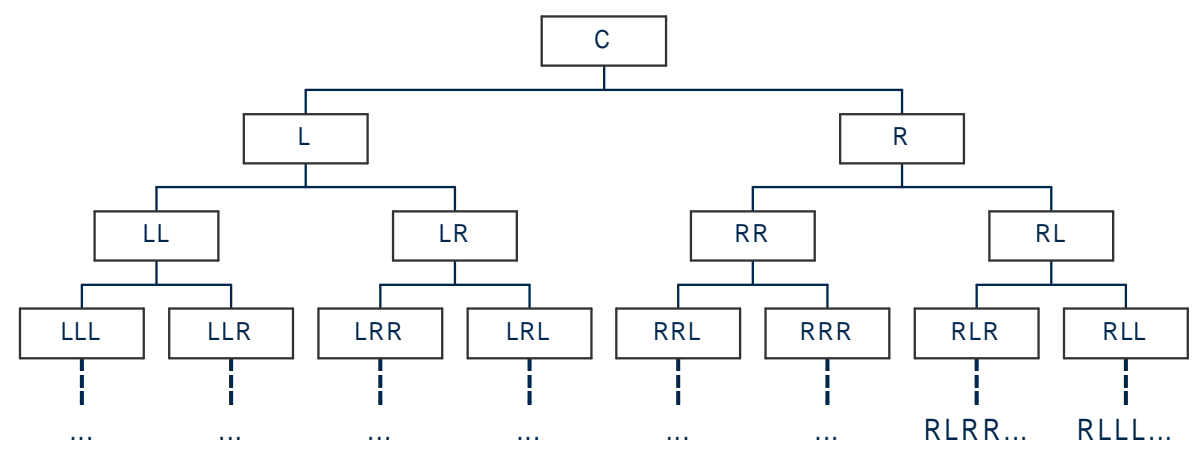

Figure 2. Tree $\mathcal{T}$ of the trajectories of unimodal maps, ordered as the topological entropy. 
So, we observed a certain monotonicity of both quantities, conductance and first non-zero eigenvalue of the laplacian, when we follow a "path" in the tree, from the first branch (corresponding to the trajectory of the fixed point, i.e., with period 1) until a final (obviously never attempted) branch (nonperiodic trajectory), observing these two values for each period, $k$, in the path. In Figure 3 and 4 is the graphic result of this observation for a family of unimodal maps and a family of bimodal maps.

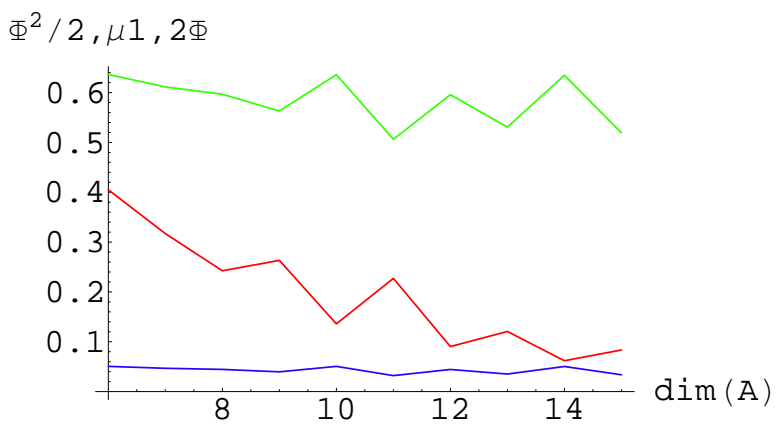

Figure 3. The higher curve is $2 \Phi$, the middle is $\mu_{1}$ and the lower is $\Phi^{2} / 2$, for the family $\left(C R L L\left(R L^{k}\right)^{\infty}\right), \quad k=1,2, \ldots$.

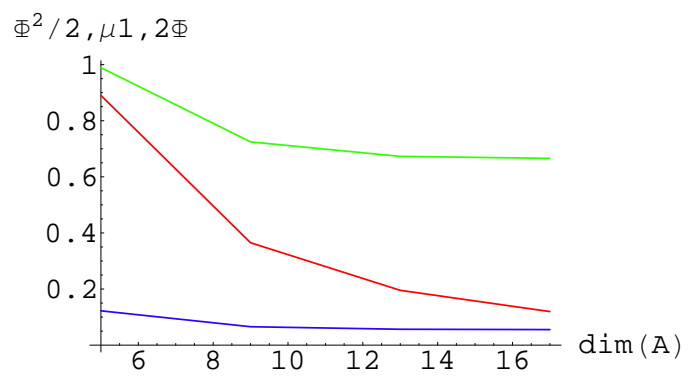

Figure 4. The higher curve is $2 \Phi$, the middle is $\mu_{1}$ and the lower is $\Phi^{2} / 2$, for the family $\left(\left(A R M^{k}\right)^{\infty},\left(B L M^{k}\right)^{\infty}\right), \quad k=1,2, \ldots$. 
The observation of these quantities for a large number of such families of maps leads us to formulate the following result, which is known and prove for the simple random walk on the graph, see Mohar ${ }^{8}$.

Theorem 2.1. Let $f$ be a map of the interval and $A_{f}$ the transition matrix associated to the Markov partition. Then the first non-zero eigenvalue $\mu_{1}\left(\Delta_{f}\right)$ of the laplacian and the conductance $\Phi_{f}$ of the Markov graph verify the following inequality

$$
2 \Phi\left(G_{f}\right) \geq \mu_{1}\left(\Delta_{f}\right) \geq \frac{\Phi\left(G_{f}\right)^{2}}{2} .
$$

This upper bound is a discrete version of the Cheeger inequality. As it was pointed out in Mohar ${ }^{9}$ it has several applications concerning the convergence rates of Markov chains to the equilibrium.

\section{Systoles in discrete dynamical systems}

We've talked about systoles in geometry and graphs. To bring this concept to the context of discrete dynamical systems we will use again the tools given by graph theory.

We can formulated the following result that establish a relation between the systole and the Betti number of the Markov graph $G_{f}$ given by $b_{f}=$ $\sum_{i, j=1} a_{i j}-n+1$, where $A_{f}=\left(a_{i, j}\right)$ is the adjacency matrix of $G_{f}$, see Balacheff ${ }^{1}$.

Theorem 3.1. Let $f$ be a map of the interval, $\left(G_{f}, w\right)$ the weighted Markov graph and $h_{\text {top }}(f)$ the topological entropy of the map $f$. Then

$$
h_{\text {top }}(f) \leq \frac{\log \left(b_{f}\right)}{\operatorname{sys}\left(G_{f}\right)} .
$$

We applied this inequality to the families we've treated before. First for unimodal family and secondly for the bimodal family. 


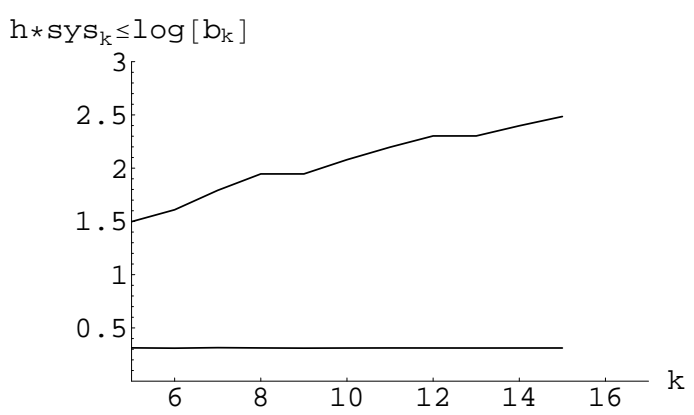

Figure 5. The higher curve is the topological entropy times the systole and the lower is the logarithm of the Betti number, for the unimodal family $\left(C R L L(R L)^{k}\right)^{\infty}, k=$ $1,2,3, \ldots$.

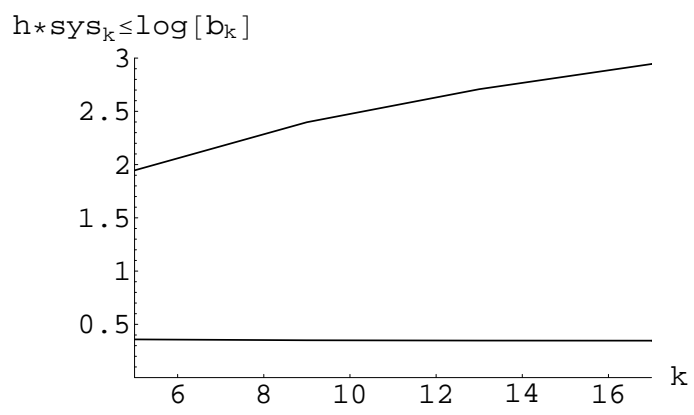

Figure 6. The higher curve is the topological entropy times the systole and the lower is the logarithm of the Betti number, for the bimodal family $\left(\left(A R M^{k}\right)^{\infty},\left(B L M^{k}\right)^{\infty}\right)$, $k=1,2,3, \ldots$

\section{Acknowledgments}

First two authors acknowledge CIMA-UE for financial support. Third author partially supported by FCT/POCTI/FEDER.

\section{References}

1. F. Balacheff, Volume entropy, systole and stable norm on graphs. math.MG/0411578.

2. M. Berger, Du côté de chez Pu, Ann. Scient. Ec. Norm. Sup. 5 (1972) pp.1-44

3. B. Bollobás. Volume estimates and rapid mixing, Flavours of Geometry. 
MSRI Publications, Vol. 31, (1997).

4. R. Brooks, P. Perry and P. Petersen. On Cheeger's inequality. Comm. Math. Helv. 68, pp. 599-621, (1993).

5. C. Grácio and J. Sousa Ramos, Spectrum of the laplacian on hyperbolic surfaces, Submitted to Grazer Mathematische Berichte.

6. S. Fernandes and J. Sousa Ramos, Conductance: from electrical networks through graphs to dynamical systems, Proceedings of NDES04 (2004).

7. J. P. Lampreia, A. Rica da Silva, J. Sousa Ramos, Subtrees of the unimodal maps tree. Boll. Un. Mat. Ital. C (6) 5 (1986), no. 1 (1987), 159-167.

8. B. Mohar, Isoperimetric inequalities, growth, and the spectrum of graphs, Linear Algebra Appl. 103, pp.119-131 (1988).

9. B. Mohar, The Laplacian Spectrum of Graphs, Graph Theory, Combinatorics and Applications, Vol.2, (1991), pp.871-898.

10. J. Milnor and W. Thurston, On iterated maps of the interval, Dynamical Systems: Proc. Univ of Maryland 1986-1987, Lecture Notes in Mathematics vol. 1342 (Springer, Berlin, New York), pp.465-563.

11. W. Parry, Intrinsic Markov chain, Trans. Amer. Math. Soc. 112 (1964), 55-65. 